\title{
AUTO-CONCEITO EM ADOLESCENTES INSTITUCIONALIZADAS: UM ESTUDO EXPLORATÓRIO
}

\author{
Eunice Magalhães ${ }^{12}$ \\ João Lopes ${ }^{2}$
}

\begin{abstract}
Resumo: A literatura sugere que o acolhimento institucional poderá ter impacto psicológico negativo na infância, nomeadamente, níveis de auto-estima reduzidos, auto-culpabilização, sentimentos de incompetência e uma percepção por parte dos jovens de uma elevada incerteza relativamente ao seu percurso de vida futuro. Neste trabalho serão apresentados resultados de um estudo comparativo ao nível do auto-conceito entre adolescentes institucionalizadas e adolescentes não institucionalizadas. O estudo envolveu 78 participantes $(100 \%$ do sexo feminino $)$ da zona Norte do país (Institucionalizadas: $\mathrm{N}=39 ; 50 \%$ ), que preencheram a Escala de Auto-conceito de Piers-Harris para Crianças. Os resultados revelaram que as adolescentes institucionalizadas apresentam níveis globais de auto-conceito mais reduzidos, tendo-se obtido resultados idênticos para algumas dimensões do auto-conceito (aspectos intelectuais, escolares e comportamentais). Contudo, outras áreas do auto-conceito parecem relativamente protegidas desta percepção menos positiva, o que poderá indiciar a presença de factores protectores. No futuro, outras dimensões de ajustamento psicológico deverão ser exploradas ao nível da investigação com adolescentes institucionalizados, numa abordagem multidimensional e se possível longitudinal.
\end{abstract}

Palavras-Chave: institucionalização, auto-conceito, adolescência.

Self-concept on institutionalized adolescents: An exploratory study (Abstract): The literature suggests that institutionalization may negatively impact adolescents who may show low self-esteem, self-blaming statements, feelings of incompetence and a perception of uncertainty concerning to the future. This paper presents results of a study of self-concept among institutionalized and non institutionalized adolescents. The study involved 78 participants $(100 \%$ females) from the north of Portugal (Institutionalized: N=39; 50\%) who completed the Scale of Self-concept of Piers-Harris for Children. The results showed that institutionalized adolescents report lower levels of global self-concept as well as low intellectual, academic and

\footnotetext{
${ }^{1}$ Contacto electrónico: nicemagalhaes@gmail.com

2 Universidade do Minho
} 
behavioral self-concept. However, other dimensions of self-concept seem fairly protected, which may show that there are protector factors. In the future research, other psychological dimensions should be explored with institutionalized adolescents, with multidimensional and longitudinal approaches.

Keywords: institutionalization, self-concept, adolescence.

A protecção na infância surge num contexto social e político de mudança relativamente ao papel da criança/jovem na sociedade, sendo que "as alterações legislativas foram determinadas, em grande parte, pela promoção da condição responsável e participativa da criança ou do jovem, enquanto sujeito dotado de autonomia e de discernimento” (Delgado, 2006, p.16). A intervenção enquadrada no sistema de promoção e protecção de crianças e jovens em perigo no contexto português contempla duas abordagens: a) medidas de intervenção em meio natural de vida (i.e., a intervenção do sistema reside na família nuclear e/ou em pessoas significativas no contexto proximal da criança); b) medidas de colocação (i.e., acolhimento familiar ou institucional) (cf. Lei n ${ }^{\circ}$ 147/99 de 1 de Setembro). O presente trabalho centra-se especificamente na medida de Acolhimento Institucional enquanto estratégia de intervenção que pressupõe o afastamento temporário da criança/jovem relativamente ao seu contexto familiar de origem. Efectivamente, a relevância desta temática no âmbito da investigação prende-se com as implicações deste afastamento na vida da criança ou jovem, na medida em que este envolve dinâmicas específicas (e.g., saída de casa, integração num contexto institucional, potencial mudança de zona de residência). Diferentes paradigmas de investigação têm caracterizado o estudo desta temática, nomeadamente, aqueles que nos anos 60 se centravam no regime institucional e na cultura informal criada pelas crianças/adolescentes e pelo corpo de técnicos da instituição de acolhimento. Já a partir da metade dos anos 70 os autores começam a focar-se na "função" da instituição e nos factores implicados nos resultados desenvolvimentais das crianças/adolescentes, e a partir da metade dos anos 90, os estudos começam a enfatizar a necessidade de planeamento e de organização das instituições de acolhimento (Clough, Bullock, \& Ward, 2006).

O decréscimo em Portugal do número de jovens institucionalizados nos últimos anos revela a maior preocupação social e política pela necessidade de "desinstitucionalização" das crianças e jovens em perigo e de uma procura activa por alternativas e projectos de vida diferenciados para estes jovens. De acordo com o Relatório de Caracterização das Crianças e Jovens em Situação de Acolhimento (Instituto de Segurança Social, 2010), a taxa de 
desinstitucionalização em Portugal foi de 21.4\% em 2009, tendo sido identificadas nesse mesmo ano 12.579 crianças e jovens em Acolhimento Institucional (menos 1.331 que em 2008). Esta preocupação pela procura activa por projectos de vida centrados no meio natural de vida decorre também dos resultados dos estudos nacionais e internacionais acerca do potencial impacto negativo da colocação das crianças em instituições (e.g., Roy, Rutter, \& Pikles, 2000; Rutter, 2000).

A experiência de acolhimento institucional representa na vida dos adolescentes um momento de mudanças significativas, sendo este momento por vezes percepcionado pelos mesmos como reflexo de abandono parental (Zem-Mascaranhas \& Dupas, 2001). A literatura sugere ainda que a integração no contexto institucional parece pautada por sentimentos de revolta por parte do adolescente, cujo desejo era o de continuar no seu contexto familiar de origem (Dunn, Culhane, \& Taussig, 2010). Além disso, os estudos salientam que a institucionalização poderá conduzir à potencial perda de relações significativas, que se reflecte na possibilidade de comprometimento desenvolvimental, na medida em que, a qualidade das relações interpessoais constitui não só um indicador de ajustamento no presente mas também de organização e qualidade das relações futuras (Martins, 2005).

A investigação internacional tem desenvolvido esforços no sentido de compreender quais as variáveis explicativas das trajectórias desenvolvimentais de jovens acolhidos institucionalmente (Rutter, 2000). Os estudos têm reportado a presença de problemas de internalização e externalização em crianças/jovens acolhidos institucionalmente quando comparados com crianças/adolescentes sem história de acolhimento institucional e a viver com os pais biológicos (Zeanah, Egger, Smyke, Nelson, Fox, Marshall, \& Guthrie, 2009). Do mesmo modo, estas crianças/jovens quando comparadas com os pares em acolhimento familiar tendem a revelar mais problemas de hiperactividade e défice de atenção em contexto de sala de aula (Roy et al., 2000). Efectivamente, estudos que analisam o funcionamento académico de crianças/jovens acolhidos institucionalmente reportam dificuldades ao nível do seu desempenho e comportamentos desadaptativos em contexto escolar (Attar-Schwartz, 2009; Trout, Hagaman, Casey, Reid, \& Epstein, 2008). Em termos de impacto psicossocial na idade adulta, estudos recentes reportam elevados níveis de perturbação psicológica (especificamente, perturbações de ansiedade, depressão, ou abuso de substâncias), sintomas traumáticos, e estilos de vinculação inseguros em indivíduos que estiveram acolhidos institucionalmente durante a infância, quando comparados com grupos normativos ou amostras da comunidade (Carr et al., 2010).

A literatura sugere que estes resultados, centrados no impacto potencialmente negativo, podem ser analisados à luz de algumas hipóteses explicativas, designadamente: 1) os padrões de qualidade dos cuidados providen- 
ciados em contexto institucional (Roy et al., 2000); 2) a própria disrupção familiar e individual prévia ao acolhimento (Rutter, 2000); 3) o nível de envolvimento e participação do jovem nas dinâmicas institucionais; e 4) as experiências de abuso prévias ao acolhimento e em contexto institucional (Carr et al., 2010). Com efeito, as próprias experiências de vitimação podem constituir um factor de risco para a presença de problemas comportamentais (Glaser, 2005; Levendosky, Okun, \& Parker, 1995; Taussig, 2002; Thompson \& Tabone, 2010), de sintomatologia internalizadora (Bolger \& Patterson, 2001) e de maior rejeição social nestes adolescentes (Erikson, Egeland, \& Pianta, 1997). Mais ainda, o maltrato prévio parece estar associado a um padrão comportamental disfuncional e não apenas a resultados comportamentais desadequados num determinado momento (Thompson \& Tabone, 2010). De salientar que este padrão comportamental externalizador parece acentuado pelo número de separações da família de origem e pelas sucessivas mudanças de instituição (Adam \& Chase-Lansdale, 2002). Além disso, ao nível do estabelecimento de relações de vinculação adaptativas, as experiências de vitimação prévias colocam constrangimentos ao desenvolvimento ajustado de relações interpessoais securizantes - decorrente, por exemplo, de percepções por parte do jovem de insegurança, medo e de que o ambiente à sua volta é perigoso (Lowenthal, 2000).

Do mesmo modo, o acolhimento institucional parece estar associado a uma percepção por parte do adolescente de incerteza relativamente ao futuro, bem como de uma reduzida controlabilidade no que concerne ao seu percurso de vida (Zem-Mascaranhas \& Dupas, 2001). Com efeito, se o processo de institucionalização depende de factores externos ao adolescente, designadamente, das decisões das entidades com competência nesta matéria, bem como da capacidade dos próprios pais em termos de reestruturação das condições de vida, o jovem poderá sentir-se desprovido de controlo sobre qualquer decisão relativa ao seu futuro (Rutter, 2000). Aliado à ausência de controlo nas suas vidas, encontra-se também um aspecto importante em termos de dinâmicas e funcionamento institucional, que está relacionado com as reduzidas oportunidades de autonomia e de tomada de decisão dos jovens neste contexto (Rutter, 2000).

Tendo em conta a complexidade inerente à adaptação dos adolescentes acolhidos importa explorar especificamente a forma como estes se percepcionam em diferentes aspectos do "self". Deste modo, o presente trabalho centrar-se-á na dimensão do auto-conceito, assumindo que esta dimensão reflecte a forma como o adolescente se percepciona em diferentes aspectos relevantes para o seu desenvolvimento (e.g., relação com outros significativos, percepção acerca do seu estatuto intelectual e social).

A literatura acerca do auto-conceito em adolescentes acolhidos é relativamente escassa e centra-se em grupos muito específicos, como por exem- 
plo, jovens delinquentes (e.g., Chassin, Presson, Young, \& Light, 1981). Efectivamente, analisando a literatura existente especificamente relativa ao constructo "auto-conceito", verificamos que o seu estudo em jovens acolhidos institucionalmente é escasso a partir dos anos 90, e constitui uma realidade quase exclusivamente norte-americana. Neste sentido, os resultados dos trabalhos publicados revelam que os jovens institucionalizados apresentam valores significativamente mais elevados na egocentricidade e mais reduzidos na auto-estima, interesse social, individuação, identificação grupal, e identificação com o professor e pares (dimensões do self avaliadas) quando comparados com adolescentes não acolhidos (Long, Zieler, \& Bankes, 1970). Do mesmo modo, adolescentes acolhidos em internamento hospitalar revelam percentagens de auto-conceito desviante mais elevadas comparativamente aos adolescentes na comunidade (Chassin, Presson, Young, \& Light, 1981). Ainda durante os anos 70, Ziegles, Balla e Watson (1972), no seu estudo com crianças e jovens norte-americanos (apenas do sexo masculino), e utilizando duas medidas de disparidade da Auto-Imagem, concluíram que as crianças e jovens institucionalizados apresentaram uma maior pontuação na medida de disparidade da auto-imagem do que os não institucionalizados, e uma menor pontuação ao nível da auto-imagem real e auto-imagem ideal. Duas décadas mais tarde, um outro estudo vem contribuir para a análise da auto-imagem com recurso a indicadores emocionais (Pasian \& Jackmin, 1999). Os resultados revelaram que as crianças acolhidas apresentaram mais indicadores emocionais na Escala de indicadores emocionais de Koppitz do que as não acolhidas (e.g., integração pobre de partes da figura). Não obstante, os autores salientam que apesar do potencial impacto negativo do acolhimento institucional a nível emocional e do auto-conceito, a qualidade das experiências em contexto de acolhimento revelou-se fundamental para a não cristalização dos processos de auto-desvalorização (Pasian \& Jackmin, 1999).

A relevância do estudo do auto-conceito não se circunscreve à percepção global mas também à exploração de dimensões específicas do auto-conceito (e.g., académico e intelectual). De facto, a literatura tem sugerido que os jovens em acolhimento experienciam uma multiplicidade de experiências potencialmente comprometedoras do seu funcionamento psicológico e social (e.g., história prévia de abuso, elevado número de mudanças de instituição de acolhimento) (Hussey \& Guo, 2002). Neste sentido, estas experiências poderão influenciar a percepção acerca de si próprios em diferentes níveis do "self" (e.g., aspectos físicos, atitudes, competências, e aceitação social) (Marsh \& Hattie, 1996). No domínio académico, os estudos têm reportado pior desempenho e reduzidas competências cognitivas em jovens acolhidos, o que poderá comprometer a sua auto-percepção ao nível dos atributos académicos e intelectuais (Sullivan, Jones, \& Mathiesen, 2010). Além disso, quando se comparam adolescentes em diferentes contextos, 
designadamente, acolhimento em contexto familiar, institucional e contexto natural de vida, o comprometimento em termos académicos e comportamentais tende a ser mais significativo no grupo de jovens que se desenvolve em contextos mais restritivos, como o caso do acolhimento institucional (Stone, 2007). Do ponto de vista social e emocional, a literatura sugere que estes jovens tendem a sentir-se mais estigmatizados e rejeitados socialmente do que os pares integrados na família nuclear, alargada ou adoptiva (Canha, 2000). Do mesmo modo, os estudos reportam níveis de auto-estima reduzidos, auto-culpabilização e sentimentos de incompetência em jovens acolhidos (Alberto, 2002). Uma vez que o sentido pessoal de identidade está tipicamente associado a um auto-conceito positivo, as dificuldades de valorização pessoal parecem influenciar a formação do auto-conceito nesta população (Lobel \& Winch, 1987).

Através desta síntese introdutória, pudemos verificar que, de um modo geral, os jovens acolhidos institucionalmente quando comparados com grupos de adolescentes na comunidade tendem a apresentar pontuações mais reduzidas nas medidas de auto-conceito utilizadas. Não obstante, analisando criticamente estes estudos verificamos que, para além destes trabalhos remontarem na sua maioria aos anos 70/80 - e, por conseguinte, a realidade do acolhimento institucional ser actualmente diferenciada - os instrumentos utilizados são muito distintos e específicos para os estudos em questão (e.g., Escala de Indicadores emocionais de Koppitz). Analisando os trabalhos realizados na última década verificamos que estes não contemplam o auto-conceito como variável resultado, centrando-se maioritariamente em aspectos de desempenho académico, funcionamento psicológico e comportamental (e.g., Trout et al., 2008).

Assim, o estudo do auto-conceito constitui-se como inovador no domínio científico nacional e internacional. O presente trabalho pretende compreender de que forma uma amostra de adolescentes acolhidas institucionalmente se diferenciam das não acolhidas ao nível do auto-conceito, através de uma análise comparativa entre os dois grupos. Com base nos resultados de estudos realizados nesta população ao nível do auto-conceito (e.g., Chassin et al., 1981), da estigmatização e rejeição social (Canha, 2000; Alberto, 2002), e naqueles que reportam um comprometimento no funcionamento emocional e comportamental nestes jovens (Rutter, 2000; Hagaman et al., 2010; Attar-Schwartz, 2009), hipotetiza-se que as adolescentes acolhidas institucionalmente apresentam pontuações de auto-conceito significativamente inferiores às não acolhidas. 


\section{Método}

\section{Participantes}

O estudo incluiu 78 participantes do sexo feminino provenientes da zona Norte do país, com idades compreendidas entre os 12 e os 17 anos $(M=15.05 ; D P=1.33)$. Trinta e nove das participantes encontravam-se acolhidas institucionalmente, distribuídas por quatro Lares de Infância e Juventude e um Centro de Acolhimento Temporário. O mesmo número de jovens não acolhidas institucionalmente encontrava-se em contexto escolar, sem história que configurasse uma situação de perigo/risco psicossocial, nem história prévia de acolhimento ${ }^{3}$.

Comparando os grupos em estudo, verificamos que a média de idade do grupo de adolescentes acolhidas $(M=15.33 ; D P=1.26)$ é superior à do grupo de não acolhidas $(M=14.79 ; D P=1.36)$, apesar de esta diferença não ser estatisticamente significativa $(\mathrm{t}(78)=-1.81, \mathrm{n} . \mathrm{s})$. Do ponto de vista da escolaridade das adolescentes, verificamos que em ambos os grupos, o $8^{\circ}$ ano é o nível de escolaridade com maior expressão - adolescentes institucionalizadas $39 \%$ e não institucionalizadas $44 \%$. Finalmente, no que se refere à categoria profissional dos progenitores, a categoria de "Operários não qualificados" é a que apresenta maior percentagem em ambos os grupos para os pais (apesar de com maior expressão no grupo de não institucionalizadas), sendo que no caso das mães, os grupos se diferenciam. No grupo de adolescentes institucionalizadas a categoria "Doméstica" é aquela que aparece com maior percentagem, já no grupo de adolescentes não acolhidas é a categoria "Operários não qualificados" (Quadro I).

A amostra de participantes em estudo foi constituída por conveniência. Foram considerados para o presente estudo adolescentes que cumprissem os seguintes critérios: 1) idade igual ou superior a 12 anos e inferior a 18 anos; 2) as adolescentes acolhidas institucionalmente teriam de se encontrar numa de duas respostas sociais em termos de promoção e protecção na infância: ou em Lares de Infância e Juventude ou em Centros de Acolhimento Temporário; 3) as adolescentes não acolhidas institucionalmente não apresentavam história prévia de acolhimento, podendo estar integradas em

\footnotetext{
${ }^{3}$ Inicialmente, a amostra era constituída por 124 adolescentes. No entanto, no sentido de emparelhar os dois grupos em termos de características sociodemográficas foram eliminados todos os adolescentes do sexo masculino $(N=38)$ uma vez que o grupo de adolescentes acolhidos se encontrava desequilibrado ao nível do sexo. Além disso, os grupos foram ainda emparelhados ao nível da idade (tendo para isso sido eliminadas 8 participantes), não havendo diferenças estatisticamente significativas nesta variável na amostra final.
} 
diferentes contextos naturais de vida (e.g., família de origem, família alargada, entre outros).

Quadro I

Caracterização das Participantes

\begin{tabular}{|c|c|c|c|}
\hline & $\begin{array}{c}\text { Adolescentes } \\
\text { institucionaliza- } \\
\text { das } \\
(N=39)\end{array}$ & $\begin{array}{c}\text { Adolescentes } \\
\text { não Institucio- } \\
\text { nalizadas } \\
(N=39)\end{array}$ & $\begin{array}{l}\text { Compara- } \\
\text { ção de gru- } \\
\text { pos }\end{array}$ \\
\hline \multirow[t]{3}{*}{ Idade } & $\begin{array}{c}M=15.33(D P= \\
1.26)\end{array}$ & $M=14.79$ & $\begin{array}{c}t(78)=-1.81, \\
p=.074\end{array}$ \\
\hline & & $(D P=1.36)$ & \\
\hline & \multicolumn{2}{|c|}{ Frequência (\%) } & \\
\hline \multicolumn{4}{|l|}{ Escolaridade $^{4}$} \\
\hline $5^{\circ}$ ano & $0(0)$ & $1(2.6)$ & \\
\hline $6^{\circ}$ ano & $8(22.2)$ & $8(20.5)$ & \\
\hline $7^{\circ}$ ano & $7(19.4)$ & $2(5.1)$ & \\
\hline $8^{\circ}$ ano & $14(38.9)$ & $17(43.6)$ & \\
\hline $9^{\circ}$ ano & $5(13.9)$ & $4(10.3)$ & \\
\hline $10^{\circ}$ ano & $1(2.8)$ & $4(10.3)$ & \\
\hline $11^{\circ}$ ano & $1(2.8)$ & $3(7.7)$ & \\
\hline \multicolumn{4}{|l|}{ Profissão da Mãe } \\
\hline Profissões Intelectuais e Científicas & $2(4.9)$ & 0 & \\
\hline Operários qualificados e serviços & 0 & $6(15.4)$ & \\
\hline Operários não qualificados & $11(26.8)$ & $20(51.3)$ & \\
\hline Reformada & $1(2.4)$ & 0 & \\
\hline Doméstica & $14(36.6)$ & $10(29.3)$ & \\
\hline \multicolumn{4}{|l|}{ Profissão do Pai } \\
\hline Operários qualificados e serviços & $3(7.3)$ & $3(10.5)$ & \\
\hline Operários não qualificados & $15(36.6)$ & $29(86.8)$ & \\
\hline Reformado & $2(4.9)$ & $1(2.6)$ & \\
\hline
\end{tabular}

4 Tendo em conta que a amplitude etária dos participantes é muito reduzida (12-17 anos) optámos por apresentar a escolaridade em termos de ano de escolaridade concluído e não de ciclo de estudos para que pudesse ser mais discriminativo e explicativo. 


\section{Medidas}

Auto-conceito. Para a avaliar o auto-conceito foi usada a Escala de Auto-conceito de Piers-Harris para Crianças (P.H.C.S.C.S. 2 - Piers \& Herzberg, 2002, adaptado por Veiga, 2006). A avaliação do auto-conceito em contexto de investigação tem assumido particular relevância ao longo das últimas décadas, constituindo-se como uma dimensão multifacetada potencialmente relacionada com o comportamento humano (Byrne, 1996; Veiga, 2006). A literatura tem demonstrado que a P.H.C.S.C.S. 2 constitui um instrumento relevante no contexto de investigação, na medida em que, não só tem contribuído para o acréscimo de conhecimento científico, como também tem sido largamente utilizada em diferentes contextos de investigação com crianças/jovens (e.g., escolar, saúde) (Wilgenbusch \& Merrel, 1999; Veiga, 2006). Além disso, este instrumento tem sido reportado como de interesse particular quando se pretende reflectir os resultados de investigação em programas de intervenção (Byrne, 1996). De facto, a escolha deste instrumento para o presente trabalho decorre dos seguintes argumentos: a) características psicométricas válidas para a população portuguesa; b) possibilidade de comparabilidade de resultados, pelo facto de se tratar de uma escala utilizada em estudos desenvolvidos internacionalmente; c) adequabilidade da escala ao contexto da presente investigação, pela sua utilização prévia em contextos de vitimação na infância.

A presente escala foi originalmente criada por Piers em 1984, sendo que a sua versão portuguesa reduzida (adaptada por Veiga, 2006) é constituída por 60 itens preenchidos de acordo com uma escala dicotómica de "sim" e "não", e sendo atribuído 1 ponto por cada resposta que vai de encontro a uma perspectiva positiva de si mesmo, e 0 quando isto não acontece. $\mathrm{O}$ tempo médio de preenchimento da presente escala é de 10 minutos. Para além da pontuação global (valores que podem variar entre 0 e 60 ), esta escala pode ser analisada de acordo com as seguintes seis componentes ou dimensões.

A dimensão "Aspecto Comportamental" refere-se à percepção da criança/adolescente acerca da adequabilidade do seu comportamento em diferentes contextos, e inclui 13 itens (e.g., "Meto-me frequentemente em sarilhos"; "Meto-me em muitas brigas"). O "Estatuto Intelectual e Escolar" envolve a percepção da criança/adolescente no que diz respeito às suas competências intelectuais e escolares, bem como acerca da forma como os outros significativos lhe atribuem competência e estatuto em termos intelectuais e escolares, sendo avaliada através de 13 itens (e.g., "Sou lento a terminar os trabalhos escolares"; "Sou um membro importante na minha turma"). No que concerne à dimensão "Aparência e Atributos físicos" esta pretende avaliar a forma como a criança/adolescente se percepciona em termos de aspecto físico e a sua satisfação com o mesmo, e inclui 8 itens (e.g., "Tenho um 
cabelo bonito"; "Tenho uma cara agradável"). A dimensão "Ansiedade" refere-se à preocupação e medo reportados pelas crianças/adolescentes relativamente a situações e contextos diários, sendo avaliada através de 8 itens (e.g., "Fico nervoso quando o professor me faz perguntas"; "Preocupo-me muito"). Os itens desta dimensão são codificados em ordem invertida, permitindo assim que uma maior pontuação nesta dimensão corresponda a menores níveis de ansiedade. A "Popularidade" refere-se à percepção da criança/adolescente acerca da sua aceitação em contexto social/grupal e à facilidade em estabelecer relações interpessoais no seu contexto diário, e inclui 10 itens (e.g., "Os meus colegas troçam de mim"; "Tenho dificuldade em fazer amigos"). Finalmente, a "Satisfação e Felicidade" refere-se à percepção global da criança/adolescente no que diz respeito às suas qualidades e a satisfação percebida face à sua vida, sendo avaliada por 8 itens (e.g., "Gostava de ser diferente daquilo que sou"; "Sou uma boa pessoa") (Veiga, 2006).

No seu estudo da versão original, Piers (1984, citado por Veiga, 2006) aplicou a escala a uma amostra de 1183 crianças, tendo encontrado uma média de 50.84 pontos $(D P=13.87)$ e uma variância explicada pelos seis factores de $42 \%$. Na versão portuguesa, a média obtida foi de 41.7 pontos $(D P=7.88)$, e a variância explicada de $34 \%$ (Veiga, 2006). No presente estudo, a média obtida foi de 41.40 pontos $(D P=8.32)$. Um outro estudo em contexto nacional testou a validade de construto deste instrumento em crianças/adolescentes vítimas de maltrato na infância, tendo obtido uma variância explicada de $37.6 \%$ para a estrutura factorial de seis dimensões (Alberto, 2006).

Relativamente à fidelidade da versão portuguesa, a escala global apresenta um coeficiente de consistência interna (Alpha de Cronbach) de.90 (.86 para os participantes do sexo masculino e de .88 para os do sexo feminino) (Veiga, 2006). Considerando as componentes da escala, Veiga (2006) refere os seguintes valores de consistência interna: "Ansiedade" (. 62), "Satisfação e Felicidade" (. 67), "Popularidade" (.70), "Aparência e Atributos físicos" (.72), "Aspecto Comportamental" (.74) e "Estatuto Intelectual e Escolar" (.75) (Veiga, 2006). No presente estudo, os valores de consistência interna obtidos foram os seguintes: Auto-conceito global (.86), "Ansiedade" (.62), "Satisfação e Felicidade" (.67), "Popularidade" (.62), "Aparência e Atributos físicos" (.50), "Aspecto Comportamental" (.70) e "Estatuto Intelectual e Escolar" (.70). Os valores de consistência interna são genericamente aceitáveis para a medida global bem como para todas as dimensões, com excepção da dimensão "Aparência e Atributos Físicos" cujo valor de alpha se revelou abaixo do expectável para ser considerado aceitável. 


\section{Procedimento}

A administração do questionário foi efectuada após autorização prévia (na modalidade escrita) por parte dos directores das instituições de acolhimento e dos respectivos responsáveis da direcção das escolas. A recolha de dados das adolescentes acolhidas institucionalmente foi efectuada nas instituições de acolhimento, sendo que os dados relativos àquelas que se encontram integradas nas suas famílias de origem foram recolhidos nos seus estabelecimentos de ensino (i.e., escolas da rede pública de ensino).

Do ponto de vista ético, foi solicitada a participação no estudo garantindo a confidencialidade dos dados bem como o direito de privacidade e anonimato quer das instituições quer das participantes. Os objectivos do trabalho foram explicitados às participantes, tendo sido referido que este estudo pretendia compreender a percepção de cada uma delas relativamente à forma como se percepcionam em determinadas dimensões, e cujos resultados seriam tratados estatisticamente e analisados apenas no âmbito do estudo em causa. No preenchimento do questionário, não foi estabelecido tempo limite, a aplicação foi colectiva, e teve uma duração aproximada de 15 minutos. A análise de dados foi efectuada com recurso ao software SPSS/PASW statistics 18 .

\section{Resultados}

Com o objectivo de avaliar se os grupos de participantes se diferenciam ao nível do auto-conceito, procedeu-se a uma análise de variância multivariada (MANOVA), tendo em conta quer o auto-conceito global quer os seus aspectos particulares, nomeadamente a ansiedade, a satisfação e a felicidade, a popularidade, a aparência e os atributos físicos, o aspecto comportamental e o estatuto intelectual e escolar. Utilizando o Traço de Pillai, verifica-se que as diferenças entre os grupos são apenas marginalmente significativas $\left(V=0.155 ; F(6,71)=2.17, p=.05 ; \eta^{2}{ }_{p}=0.155\right)$ (Cohen, 1992). Especificamente, analisando comparativamente os dois grupos ao nível do auto-conceito global, as adolescentes acolhidas percepcionam-se menos positivamente $(M=37.41 ; D P=9.04)$ que as não acolhidas $(M=43.36$; $D P=6.97)\left(F(1,76)=10.60 ; p<.01 ; \eta^{2}{ }_{p}=0.122\right.$; I.C. a 95\% ] 2.31; 9.39 [ $)$. Resultados semelhantes verificam-se em algumas dimensões do auto-conceito. As adolescentes acolhidas institucionalmente percepcionam-se menos positivamente ao nível dos seus comportamentos, considerando-os menos adaptativos do que as não acolhidas ("Aspecto Comportamental" $F$ $(1,76)=8.89, \mathrm{p}<.01 ; \eta^{2} \mathrm{p}=0.115 ;$ I.C. a $95 \%$ ]0.55; 2.74 [). Do mesmo modo, estas adolescentes revelam uma percepção mais negativa acerca das suas competências e estatuto intelectual ("Estatuto Intelectual e Escolar" (F (1, $76)=6.30, \mathrm{p}<.05 ; \eta^{2} \mathrm{p}=0.077$; I.C. a 95\% ]0.31; 2.71[), e reportam estar 
significativamente menos satisfeitas e felizes do que as não acolhidas ("Satisfação e Felicidade" $\left(F(1,76)=6.06, \mathrm{p}<.05 ; \eta^{2} \mathrm{p}=0.074\right.$; I.C. a 95\% ]0.18; 1.67[) (cf. Quadro 2). Não foram encontradas diferenças estatisticamente significativas nas dimensões "Popularidade", "Aparência e Atributos Físicos" e "Ansiedade" entre os grupos.

Quadro II.Média e Desvio-Padrão das adolescentes institucionalizadas e não institucionalizadas ao nível das subescalas do auto-conceito

\begin{tabular}{lllll}
\hline $\begin{array}{c}\text { Dimensões de Auto-conceito } \\
\text { (amplitude da escala de resposta) }\end{array}$ & \multicolumn{1}{c}{ Grupo } & $M$ & $D P$ & $F$ \\
\hline \multirow{2}{*}{ Aspecto Comportamental (0-13) } & Não Institucionalizadas & 10.49 & 2.34 & $8.89^{* *}$ \\
& Institucionalizadas & 8.85 & 2.52 & \\
Estatuto Intelectual e Escolar (0-13) & Não Institucionalizadas & 9.49 & 1.99 & $6.30^{*}$ \\
& Institucionalizadas & 7.97 & 3.20 & \\
Satisfação e Felicidade (0-8) & Não Institucionalizadas & 6.72 & 1.41 & $6.06^{*}$ \\
& Institucionalizadas & 5.79 & 1.87 & \\
Ansiedade (0-8) & Não Institucionalizadas & 3.79 & 2.14 & 3.01 \\
& Institucionalizadas & 3.03 & 1.76 & \\
Popularidade (0-10) & Não Institucionalizadas & 7.82 & 1.90 & 3.20 \\
& Institucionalizadas & 7.03 & 2.02 & \\
Aparência e Atributos físicos (0-8) & Não Institucionalizadas & 5.05 & 1.36 & 0.62 \\
& Institucionalizadas & 4.74 & 2.04 \\
\hline
\end{tabular}

${ }^{*} p<.05 ; * * p<.01$

\section{Discussão}

O objectivo do presente trabalho passou por analisar a concepção que um grupo de adolescentes acolhidas institucionalmente tem acerca de si, comparativamente com um grupo de adolescentes não acolhidas e não referenciadas como apresentando qualquer risco psicossocial.

Os resultados confirmam a hipótese de que as adolescentes acolhidas institucionalmente apresentam pontuações de auto-conceito significativamente inferiores às não acolhidas. Efectivamente, foram encontradas diferenças significativas entre os dois grupos de adolescentes, ao nível da pontuação global da escala de auto-conceito, bem como em determinadas dimensões do constructo. Assim, globalmente, as adolescentes acolhidas institucionalmente tendem a apresentar um conceito de si mais depreciado do que as não institucionalizadas. De facto, se tivermos como valor de referência a média global obtida nos estudos de validação do instrumento em 
contexto nacional $(M=41.7)$, verificamos que a média das adolescentes acolhidas institucionalmente se encontra abaixo desse valor $(M=37.41)$ e que o grupo de comparação apresentou uma média global superior $(M=$ 43.36).

Especificamente, verifica-se que as diferenças entre os dois grupos se reportam às dimensões "Aspecto Comportamental", "Estatuto Intelectual e Escolar" e "Satisfação e Felicidade". Contudo, é necessário realçar que, nas dimensões "Aspecto Comportamental" e "Estatuto Intelectual e Escolar", os valores médios obtidos pelas adolescentes institucionalizadas se situam acima do valor médio possível de 7.5 pontos (os valores podem variar entre $0 \mathrm{e}$ 13). Do mesmo modo, para a dimensão de "Satisfação e Felicidade", a média das adolescentes institucionalizadas é superior a 4 pontos num intervalo possível de 0 a 8 . Assim, embora existam diferenças significativas entre os grupos, deve notar-se que estas diferenças se verificam num quadro em que um dos grupos (participantes institucionalizadas) está na média dos valores possíveis e o outro grupo se encontra acima da média, não podendo por isso o grupo das adolescentes institucionalizadas ser considerado deficitário a este nível. Deste modo, apesar de as situações de vitimação ou perigo experienciadas por menores institucionalizados constituírem potenciais factores de risco para a manifestação de dificuldades ao nível emocional, comportamental, e académico (Hagaman et al., 2010; Taussig, 2002; Thompson \& Tabone, 2010), tais riscos parecem minimizados em pelo menos algumas das dimensões do auto-conceito avaliadas neste estudo, no grupo de participantes institucionalizadas.

Do mesmo modo, no que diz respeito às dimensões Aparência e Atributos Físicos, Popularidade, e Ansiedade não foram encontradas diferenças significativas entre os grupos. Assim, apesar de diversos estudos apontarem para o comprometimento de vínculos afectivos estáveis nesta população (e. g. Martins, 2005), estes resultados poderão indicar que, do ponto de vista das relações interpessoais ("Popularidade"), estas adolescentes não se percepcionam necessariamente como menos aceites/integradas do que o grupo de comparação neste estudo. Com efeito, estas adolescentes acolhidas institucionalmente, apesar de se revelarem menos satisfeitas ao nível comportamental e intelectual do que o grupo de não acolhidas, parecem sentir-se satisfeitas com a sua rede de suporte social/pares e apresentam uma percepção positiva da sua capacidade de estabelecer relações interpessoais adaptativas (Veiga, 2006).

Estes resultados são inesperados, podendo a ausência de comprometimento do auto-conceito neste grupo de adolescentes acolhidas estar relacionada com factores protectores individuais, institucionais (e.g., rigor e regras da instituição) ou relacionais (e.g., contacto de suporte com técnicos, e com a família de origem) (Zem-Mascaranhas \& Dupas, 2001). Poderemos 
ainda hipotetizar que estas adolescentes possam ter construído projectos de vida pessoais diferenciados, que possam sentir-se seguras e protegidas em contexto institucional, e que tal possa também resultar numa apreciação menos negativa acerca de si. Por outro lado, é possível que também o grupo de adolescentes não acolhidas seja um grupo caracterizado por alguma especificidade, uma vez que apresenta uma média global elevada $(M=43.36)$ e acima da média obtida na validação da escala para o contexto português ( $M=$ 41.70). Finalmente, é possível que as adolescentes em ambos os grupos possam ter respondido de acordo com alguma desejabilidade social, o que poderá explicar as pontuações tendencialmente elevadas na escala.

\section{Limitações e direcções futuras}

Apesar de os resultados obtidos parecerem relevantes e indicarem a necessidade de mais investigação e exploração desta temática, importa notar algumas limitações: 1) o reduzido número de adolescentes participantes no estudo, e o facto de serem todas do sexo feminino. Com efeito, as dificuldades de acesso, quer às instituições de acolhimento, quer aos estabelecimentos de ensino, decorrentes quer da compreensível protecção das menores quer de uma significativa burocratização do processo, tornam a possibilidade de investigação neste domínio mais morosa e tornam menos provável a constituição de amostras representativas; 2) ausência de outras fontes de informação para além das adolescentes. Para uma melhor compreensão do fenómeno poderia ser relevante a exploração de outras fontes de informação significativas, no sentido de compreender de forma mais abrangente o ajustamento psicológico, social e emocional destas adolescentes; 3) limitações do design do estudo, nomeadamente a avaliação num único momento, e a ausência de variáveis relacionadas com o processo de acolhimento (e.g., tempo de acolhimento, características da instituição), limitando a compreensão/discussão dos resultados.

Tendo em consideração os dados disponíveis na literatura, bem como os resultados do presente trabalho, parece essencial que a investigação futura neste domínio inclua abordagens multidimensionais e integrativas. De facto, a investigação no futuro deverá incluir outros indicadores de ajustamento psicológico (e.g., emocional, social, comportamental) que permitam compreender de forma multidimensional o bem-estar psicossocial dos jovens em acolhimento (Earls \& Carlson, 2001; Winkel, Saeger, \& Evans, 2009). Do mesmo modo, importa identificar os mecanismos subjacentes ao processo de adaptação ao contexto de acolhimento institucional e as condições facilitadoras deste processo (e.g., características do ambiente institucional, qualidade da relação com os técnicos da instituição, suporte social/institucional) (Sto- 
ne, 2007; Trout et al., 2008). Em termos de design metodológico, o esforço deverá ir no sentido da conciliação de modalidades de investigação, de cariz longitudinal, transversal, quantitativo ou qualitativo (e.g., Christiansen, Havik, $\&$ Anderssen, 2010). Finalmente, importa desenvolver um esforço maior no que concerne à teorização desta problemática, uma vez que até ao momento esta é ainda escassa e dispersa (Clough, Bullock, \& Ward, 2006).

Em suma, torna-se assim essencial que, no futuro, possam ser testados modelos explicativos da percepção mais negativa destas adolescentes acerca do seu auto-conceito global e, em particular, o comportamental, intelectual e a satisfação pessoal. De facto, é importante identificar mecanismos protectores que possam ser explicativos de um desenvolvimento e identidade pessoal ajustados em jovens em acolhimento institucional comparativamente a jovens em contexto natural de vida (Hattie \& Marsh, 1996).

\section{Conclusão}

O presente estudo tinha como objectivo compreender se as adolescentes acolhidas institucionalmente se diferenciam das não acolhidas ao nível do auto-conceito. Os resultados revelam que as jovens acolhidas pontuam significativamente abaixo do grupo de comparação (adolescentes não-institucionalizadas sem referenciação de risco psicossocial) no auto-conceito global, bem como nos domínios comportamental, intelectual e de satisfação e felicidade, mas não abaixo dos valores médios da escala. Dado que estes resultados só parcialmente corroboram os resultados de estudos anteriores, espera-se que no futuro seja possível esclarecer mais cabalmente esta questão, nomeadamente através de trabalhos que possam ultrapassar algumas das limitações metodológicas atrás enunciadas (e. g. número reduzido de participantes dadas as dificuldades de acesso às instituições).

\section{Referências}

Adam, E., \& Chase-Lansdale, P. L. (2002). Home sweet homes: parental separations, residential moves, and adjustment problems in low-income adolescent girls. Developmental Psychology, 38(5), 792-805.

Alberto, I. (2002). "Como pássaros em gaiolas"? Reflexões em torno da institucionalização de menores em risco. In C. Machado \& R. Gonçalves (Eds), Violência e vitimas de crimes. Volume 2 - Crianças (pp. 223-244). Coimbra: Quarteto Editora.

Alberto, I. (2006). Maltrato e trauma na infância. Coimbra: Edições Almedina.

Attar-Schwartz, S. (2009). School functioning of children in residential care: the contributions of multilevel correlates. Child Abuse \& Neglect, 33, 429-440. 
Bolger, K., \& Patterson, C. (2001). Pathways from child maltreatment to internalizing problems: perceptions of control as mediators and moderators. Development and Psychopathology, 13, 913-940.

Bolger, K., Patterson, C., \& Kupersmidt, J. (1998). Peer relationships and self-esteem among children who has been maltreated. Child Development, 69(4), 1171-1197.

Byrne, B. (1996). Measuring self-concept across the life span. Washington: American Psychological Association.

Canha, J. (2000). Criança maltratada. O papel de uma pessoa de referência na sua recuperação. Estudo prospectivo de 5 anos. Coimbra: Quarteto.

Carr, A., Dooley, B., Fitzpatrcik, M., Flanagan, E., Flanagan-Howard, R., Tierney, K., White, M., Daly, M., \& Egan, J. (2010). Adult adjustment of survivors of institutional child abuse in Ireland. Child Abuse \& Neglect, 34, 477-489.

Clough, R., Bullock, R., \& Ward, A. (2006). What works in residential child care. A review of research evidence and the practical considerations. London: National Children's Bureau

Chassin, L., Presson, C., Young, R., \& Light, R. (1981). Self-Concepts of Institutionalized Adolescents: A Framework for Conceptualizing Labeling Effects. Journal of Abnormal Psychology, 90(2), 143-151.

Christiansen, O., Havik, T., \& Anderssen, N. (2010). Arranging stability for children in long-term out-of-home care. Children and Youth Services Review, 32, $913-$ -921 .

Cohen, J (1992). A power primer. Psychological Bulletin, 112, 155-159.

Delgado, P. (2006). Os direitos da Criança. Da participação à responsabilidade. $O$ sistema de protecção e educação das crianças e jovens. Maia: Profedições.

Dunn, D., Culhane, S., \& Taussig, H. (2010). Children's appraisals of their experiences in out-of-home care. Children and Youth Services Review, 32, 1324$-1330$.

Earls, F., \& Carlson, M. (2001). The social ecology of child health and well-being. Annual Review of Public Health, 22, 143-166.

Erikson, M., Egeland, B., \& Pianta, R (1997). Effects of maltreatment on the development of young children. In D. Cicchetti \& V. Carlson (Eds), Child Maltreatment: theory and research on the causes and consequences of child abuse and neglect (pp. 647-684). United Kingdom: Cambridge University Press

Glaser, D. (2005). Child maltreatment. Psychiatry,4(7), 53-57.

Hagaman, J., Trout, A., Chmelka, M., Thompson, R., \& Reid, R. (2010). Risk profiles of children entering residential care: a cluster analysis. Journal of Child and Family Studies, 19, 525-535.

Hawkins-Rodgers, Y. (2007). Adolescents adjusting to a group home environment: A residential care model of re-organizing attachment behavior and building resiliency. Children and Youth Services Review, 29, 1131-1141.

Hattie, J., \& Marsh, H. (1996). Future directions in self-concept research. In B. Bracken (Ed) Handbook of self-concept: Developmental, social, and clinical considerations (pp. 421-462). New York: John Wiley \& Sons, Inc. 
Higgins, D., \& McCabe, M. (2001). Multiple forms of child abuse and neglect: adult retrospective reports. Aggression and Violent Behavior, 6, 547-578.

Hobbs, G., Hobbs, C., \& Wynne, J. (1999). Abuse of children in foster and residential care. Child Abuse \& Neglect, 23(12), 1239-1252.

Hussey, D., \& Guo, S. (2002). Profile characteristics and behavioral change trajectories of young residential children. Journal of Child and Family Studies, 11 (4), 401-410.

Instituto de Segurança Social (2010). Plano de Intervenção Imediata - Relatório de Caracterização das Crianças e Jovens em Situação de Acolhimento em 2009. Retirado do website da Casa Pia: http://www.casapia.pt/LinkClick.aspx? fileticket $=$ xsCRqWzcVyQ\%3D\&tabid=316\&language $=$ pt-PT.

Levendosky, A., Okun, A., \& Parker, J. (1995). Depression and maltreatment as predictors of social competence and social problem-solving skills in school-age children. Child Abuse \& Neglect, 19(10),1183-1195.

Lobel, T., \& Winch, G., (1987). Psychosocial development, self-concept and gender. The Journal of Genetic Psychology, 149 (3), 405-411.

Long, B., Zieler, R., \& Bankes, J. (1970). Self-other orientations of institutionalized behavior-problem adolescents. Journal of Consulting and Clinical Psychology, 34(1), 43-47.

Lowenthal, B. (2000). Child maltreatment: effects on development and learning. Educational Resources Information Centers, 365-371.

Magalhães, T. (2004). Maus-tratos em crianças e jovens. Guia prático para profissionais. Coimbra: Quarteto Editores.

Maroco, J. (2010). Análise estatística com o PASW Statistics (ex-spss). Pêro Pinheiro: Report Number, Análise e Gestão de Informação, Lda.

Martins, P. (2005). O desenvolvimento pessoal e social da criança em contexto de vida institucional - elementos para uma análise da ecologia da interpessoalidade. Retirado da Universidade do Minho, Repositorium dos Serviços de Documentação: https://repositorium.sdum.uminho.pt/handle/1822/6750

Marsh, H. W., \& Hattie, J. (1996). Theoretical perspectives on the structure of self-concept. In B. A. Bracken (Ed), Handbook of self-concept: Developmental, social and clinical considerations (pp. 38-90). New York: John Wiley \& Sons, Inc.

Myers, J., O’Neill, T., \& Jones, J. (1999). Preventing institutional abuse: an exploration of children's rights, needs and participation in residential care. In C. Adams, A. Ashworth, E. Blynth, H. et al. (Eds), Children, Child abuse and child protection. Placing children centrally. The violence against children study group (pp. 193-210). New York: John Wiley \& Sons.

Pasian, S., \& Jackmin, A. (1999). O auto-retrato em crianças institucionalizadas. Paidéia, 50-60.

Raymond, I., \& Heseltine, K. (2008). What does it mean to be an adult? Perceptions of young men in residential care. Child Youth Care Forum, 37, 197-208.

Roy, P., Rutter, M., \& Pikles, A. (2000). Institutional care: risk from family background or pattern of rearing? Journal of Child Psychology and Psychiatry, 41(2), 139-149. 
Rutter, M. (2000). Children in substitute care: Some conceptual considerations and research implications. Children and Youth Services, 22(9), 685-703.

Stone, S. (2007). Child maltreatment, out-of-home placement and academic vulnerability: A fifteen-year review of evidence and future directions. Children and Youth Services Review, 29, 139-161.

Sullivan, M., Jones, L., \& Mathiesen, S (2010). School change, academic progress, and behavior problems in a sample of foster youth. Children and Youth Services Review, 32(2), 164-170.

Taussig, H. (2002). Risk behaviors in maltreated youth placed in foster care: a longitudinal study of protective and vulnerability factors. Child Abuse \& Neglect, 26, 1179-1199.

Thompson, R., \& Tabone, J. (2010). The impact of early alleged maltreatment on behavioral trajectories. Child abuse \& Neglect, 34, 907-916.

Trout, A., Hagaman, J., Casey, K., Reid, R., \& Epstein, M. (2008). The academic status of children and youth in out-of-home care: A review of the literature. Children and Youth Services Review, 30, 979-994.

Wilgenbusch, T., \& Merrell, K. W. (1999). Gender differences in self-concept among children and adolescents: a meta-analysis of multidimensional studies. School Psychology Quarterly, 14, 101-20.

Winkel, G., Saegert, S., \& Evans, G. (2009). An ecological perspective on theory, methods and analysis in environmental psychology: advances and challenges. Journal of Environmental Psychology, 29, 318-328.

Veiga, F. H. (2006). Uma nova versão da escala de autoconceito: Piers-Harris Children's Self-Concept Scale (PHCSCS-2). Revista de Educação e Psicologia, 2, 39-48.

Vilaverde, M. (2000). Factores de risco e factores protectores em crianças vítimas de maus-tratos a viver em Instituições. Dissertação de Mestrado não publicada. Instituto de Educação e Psicologia, Universidade do Minho: Braga

Zeanah, C., Egger, H., Smyke, A., Nelson, C., Fox, N., Marshall, P., \& Guthrie, D. (2009). Institutional rearing and psychiatric disorders in Romanian preschool children. American Journal of Psychiatry, 166, 777-785.

Zem-Mascarenhas, S., \& Dupas, G. (2001). Conhecendo a experiência de crianças institucionalizadas. Revista da Escola de Enfermagem USP, 35(4), 413-9.

Ziegles, E., Balla, D., \& Watson, N. (1972). Developmental and experiential determinants of self-image disparity in institutionalized and noninstitutionalized retarded and normal children. Journal of Personality and Social Psychology, 23(1), 81-87. 